\title{
Lead levels in human placentae from normal and malformed births
}

\author{
D. G. WIBBERlEY, A. K. KHERA, J. H. EDWARDS, AND D. I. RUSHTON
}

From the Department of Pharmacy, University of Aston in Birmingham, and Birmingham Maternity Hospital and University of Birmingham, Birmingham

SUMmaRY Placental lead levels were studied in a series of Birmingham births classified by stillbirth, neonatal death, or survival beyond one week. There was an appreciable range of lead levels even in normal births $(0 \cdot 15-3 \cdot 56 \mu \mathrm{g} / \mathrm{g})$ but nevertheless average results showed a pronounced excess of lead in those who failed to survive both birth and the neonatal period. There was no association of placental lead with impaired birthweight among survivors but, in common with other authors, we noted a seasonal variation. The placentae from Indian women had similar lead levels to those from European women and lower values were found in the normal sibs of stillbirths and neonatal deaths. The possibility is discussed that under conditions of impaired fetal health in late pregnancy the placenta may concentrate lead.

The possibility exists that current environmental levels of heavy metals such as lead, cadmium, and mercury may present a hazard to the fetus. It is undoubtedly true that exposure of the mother to abnormally high levels of any of these metals can result in fetal death or abnormality. The best documented cases are those associated with mercury, and in particular the Minnemata Bay (Matsumota et al., 1965) and Iraq incidents (Bakir et al., 1973), but in addition several workers (Cantarow and Trumper, 1944) have shown the increased liability to abortion of women employed in industries with a high exposure to lead. The presence of lead in human fetal tissues has been reported and high levels of lead in bone have been reported in two studies of stillbirths (Lanzola et al., 1973).

All three metals have been shown to be capable of crossing the placental barrier in animal studies and to cause fetal malformations and deaths (Ferm and Carpenter, 1967), and both synergistic and antagonistic reactions of lead (Ferm, 1969) and cadmium are known (Holmberg and Ferm, 1969). In view of such evidence it seemed to us that if current environmental levels of these heavy metals is in any way responsible, or partly responsible, for fetal abnormality then one would expect a correlation between the heavy metal level in placental tissue and some malformations. To examine this assumption we have
Table 1 Reported metal levels in normal human placentae

\begin{tabular}{llllll}
\hline Author & Country & Year & \multicolumn{3}{c}{ Metal level $(\mu g / g$ fresh wt). } \\
\cline { 3 - 6 } & & & $P b$ & $C d$ & $H g$ \\
\hline Baglan et al. & U.S.A. & 1974 & 0.30 & 0.017 & - \\
Collucci et al. & U.S.A. & 1973 & 0.96 & 0.068 & - \\
Einbrodt et al. & Germany & 1973 & 0.56 & - & - \\
Horiuchi et al. & Japan & 1959 & 0.57 & - & - \\
Suzuki et al. & Japan & 1971 & - & - & 0.071 \\
Thieme et al. & Germany & 1974 & 0.40 & - & - \\
Thürauf et al. & Germany & 1975 & - & 0.07 & - \\
\hline
\end{tabular}

embarked upon a detailed analysis of heavy metals in the placentae of stillbirths, normal births, and abnormal births. There are relatively few published reports (Table 1), and no previous workers have attempted this type of correlation. The present report describes our initial findings with respect to lead.

\section{Materials and methods}

Placental samples from births at the Birmingham Maternity Hospital have been stored at $-20^{\circ} \mathrm{C}$ for the past six years. In the present work 1971 samples were studied from the 1st week of each month and it was shown that the moisture levels (mean level $79.6 \%$ ) compared favourably with those of fresh samples. $(80 \%)$.

Placental samples were obtained from:

(1) All neonatal deaths and stillborn births. 
(2) The next two normal births whose mothers did not have Indian names.

(3) The next birth to a mother with an Indian name.

(4) Placentae from sibs of all those births were then obtained using hospital numbers of mothers on an ordered computer listing which printed out only women who delivered at the hospital more than once since 1970.

(5) Finally, a series of 24 premature normal births (less than $2.5 \mathrm{~kg}$ ) was defined by a consecutive series with non-Indian names in 1971.

The samples were thawed, excessive blood removed, the tissue dissolved in Soluene, and the lead estimated by flameless atomic absorption spectroscopy. We have described our solubilisation procedure (Barlow and Khera, 1975) and the problems concerning trace metal analyses in analytical papers (Khera and Wibberley, 1976). Results have been checked by double blind experiments in our laboratories and by assays in other laboratories by alternative procedures. Extremely high attention to environmental contamination was required to ensure reproducible results.

\section{Results}

The lead levels in 126 analyses of placental samples are recorded in Tables 2-9. There is an appreciable variation between the lead levels in samples from normal births to non-Indian women whom we will

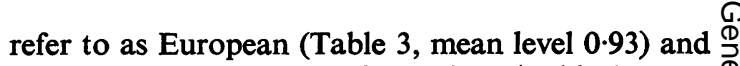
those in samples from malformations (Table 4, mean $\stackrel{\mathscr{P}}{\rightarrow}$ level 1.49), neonatal deaths (Table 5 , mean value $\vec{F}$ $1 \cdot 73$ ), or stillbirths (Table 6 , mean value $1 \cdot 45$ ). There $\stackrel{5}{+}$ was no clear relation with cause of death; in fact the three highest values included the rare condition of $\frac{\bar{D}}{\bar{N}}$ trisomy 18 (about 1 in 3000 births), a ring D chromo- $\frac{N}{D}$ some, and a congenital cerebral tumour: both the $\stackrel{\varnothing}{\propto}$ latter conditions are of exceptional rarity (less than $\%$ 1 in 50000 births).

Another group of samples were those from Asian mothers from the Indian subcontinent (i.e. India, $\vec{\omega}$ Pakistan, and Bangladesh) and these showed average $\stackrel{\circ}{S}$ levels of $1.13 \mu \mathrm{g} / \mathrm{g}$. Our 1971 results also appear to show a seasonal variation in lead levels in normal births with the lowest levels occurring in May for the $\vec{i}$ European births and in September for the Asian $\omega$ births. Dawson et al. (1968) and Baglan et al. (1974) have both reported seasonal variations with minimum 을 levels in summer months. If placental lead is solely an indicator of maternal exposure then one would have $\vec{O}$ expected sibs to have closely similar levels. This has not so far proved to be the case (Table 7).

In order to evaluate the possible contribution of $\stackrel{\mathbb{\Phi}}{\stackrel{\mathscr{S}}{-}}$ low birthweight to both high lead levels and still- $\overrightarrow{0}$ birth or neonatal death, a series of placentae from. premature European babies was then studied (Table $\square$ 8). No correlation was found between low birthweight for these normal births and high mean lead values. The mean values for the various categories are

Table 2 Lead in Indian placental samples from normal birth

\begin{tabular}{|c|c|c|c|c|c|c|c|}
\hline Delivery data & $\begin{array}{l}\text { Mother's age } \\
(y)\end{array}$ & $\begin{array}{l}\text { Gestation period } \\
(w)\end{array}$ & $\begin{array}{l}\text { Mother's weight } \\
(k g)\end{array}$ & $\begin{array}{l}\text { Child's weight } \\
(k g)\end{array}$ & Sex of child & $\begin{array}{l}\text { Social class of } \\
\text { father }\end{array}$ & $\begin{array}{l}\mathrm{Pb}(\mu \mathrm{g} / \mathrm{g} \text { fresh } \\
\text { wt basis) }\end{array}$ \\
\hline $\begin{array}{l}\text { January } \\
\text { January } \\
\text { January } \\
\text { January } \\
\text { January } \\
\text { January } \\
\text { January } \\
\text { May } \\
\text { May } \\
\text { May } \\
\text { May } \\
\text { May } \\
\text { May } \\
\text { May } \\
\text { September } \\
\text { September } \\
\text { September } \\
\text { September } \\
\text { September } \\
\text { September } \\
\text { September }\end{array}$ & $\begin{array}{l}20 \\
28 \\
27 \\
36 \\
21 \\
37 \\
39 \\
26 \\
25 \\
26 \\
28 \\
20 \\
20 \\
26 \\
18 \\
34 \\
32 \\
24 \\
28 \\
19 \\
28\end{array}$ & $\begin{array}{l}40 \\
36 \\
39 \\
38 \\
40 \\
37 \\
42 \\
40 \\
33 \\
40 \\
36 \\
40 \\
42 \\
40 \\
38 \\
41 \\
40 \\
39 \\
28\end{array}$ & $\begin{array}{l}56 \cdot 5 \\
58 \cdot 1 \\
63 \cdot 5 \\
50 \cdot 5 \\
78 \cdot 0 \\
77 \cdot 5 \\
52 \cdot 2 \\
98 \cdot 0 \\
61 \cdot 7 \\
48 \cdot 5 \\
45 \cdot 5 \\
85 \cdot 0 \\
47 \cdot 0 \\
69 \cdot 0 \\
48 \cdot 5 \\
76 \cdot 0 \\
61 \cdot 0 \\
53 \cdot 0 \\
57 \cdot 5\end{array}$ & $\begin{array}{l}2 \cdot 18 \\
3 \cdot 69 \\
3 \cdot 14 \\
3 \cdot 64 \\
3 \cdot 46 \\
3 \cdot 50 \\
3 \cdot 26 \\
- \\
3 \cdot 54 \\
2 \cdot 58 \\
3 \cdot 44 \\
2 \cdot 78 \\
3 \cdot 76 \\
3 \cdot 45 \\
3 \cdot 24 \\
3 \cdot 86 \\
4 \cdot 11 \\
3.66 \\
2 \cdot 58 \\
3.66\end{array}$ & $\begin{array}{l}\mathbf{M} \\
\mathbf{M} \\
\mathbf{M} \\
\mathbf{F} \\
\mathbf{M} \\
\mathbf{M} \\
\mathbf{F} \\
\mathbf{F} \\
\mathbf{M} \\
\mathbf{F} \\
\mathbf{M} \\
\mathbf{F} \\
\mathbf{M} \\
\mathbf{F} \\
\mathbf{M} \\
\mathbf{F} \\
\mathbf{M} \\
\mathbf{F} \\
\mathbf{F}\end{array}$ & $\begin{array}{l}3 \\
4 \\
3 \\
5 \\
3 \\
4 \\
4 \\
4 \\
3 \\
3 \\
4 \\
3 \\
3 \\
3 \\
4 \\
4 \\
5 \\
4 \\
4\end{array}$ & $\begin{array}{l}1.33 \\
0.87 \\
0.73 \\
1.20 \\
1.13 \\
1.27 \\
1.00 \\
2.16 \\
0.86 \\
1.37 \\
3.56 \\
1.25 \\
0.47 \\
1.46 \\
0.52 \\
0.99 \\
0.53 \\
0.53 \\
0.75 \\
0.97 \\
0.76\end{array}$ \\
\hline Arith. mean & $26 \cdot 8$ & $38 \cdot 9$ & $62 \cdot 5$ & $3 \cdot 34$ & - & $3 \cdot 7$ & $1.13 \pm 0.68$ \\
\hline
\end{tabular}

Range 0.47-3.56 Pb $\mu \mathrm{g} / \mathrm{g}$ fresh wt basis Arith. mean for Jan. 1.08 $\mu \mathrm{g} / \mathrm{g}$ fresh wt basis May $1.59 \mu \mathrm{g} / \mathrm{g}$ fresh wt basis $\longrightarrow=$ no information. Sept. $0.72 \mu \mathrm{g} / \mathrm{g}$ fresh wt basis 
Table 3 Lead in European placental samples from normal births

\begin{tabular}{|c|c|c|c|c|c|c|c|}
\hline Delivery data & $\begin{array}{l}\text { Mother's age } \\
(y)\end{array}$ & $\begin{array}{l}\text { Gestation } \\
\text { period }(w)\end{array}$ & $\begin{array}{l}\text { Mother's } \\
\text { weight }(k g)\end{array}$ & $\begin{array}{l}\text { Child's weight } \\
(k g)\end{array}$ & Sex of child & $\begin{array}{l}\text { Social class } \\
\text { of father }\end{array}$ & $\begin{array}{l}\mathrm{Pb}(\mu \mathrm{g} / \mathrm{g} \text { fresh } \\
\text { wt basis })\end{array}$ \\
\hline $\begin{array}{l}\text { January } \\
\text { January } \\
\text { January } \\
\text { January } \\
\text { January } \\
\text { January } \\
\text { May } \\
\text { May } \\
\text { May } \\
\text { May } \\
\text { May } \\
\text { May } \\
\text { May } \\
\text { May } \\
\text { May } \\
\text { May } \\
\text { May } \\
\text { May } \\
\text { September } \\
\text { September } \\
\text { September } \\
\text { September } \\
\text { September } \\
\text { September }\end{array}$ & $\begin{array}{l}16 \\
33 \\
24 \\
21 \\
32 \\
32 \\
38 \\
32 \\
40 \\
32 \\
33 \\
33 \\
39 \\
35 \\
30 \\
23 \\
20 \\
22 \\
28 \\
33 \\
24 \\
25 \\
22 \\
27\end{array}$ & $\begin{array}{l}41 \\
40 \\
40 \\
40 \\
42 \\
42 \\
41 \\
40 \\
38 \\
42 \\
37 \\
42 \\
38 \\
39 \\
38 \\
39 \\
41 \\
41 \\
41 \\
41 \\
41 \\
43 \\
38\end{array}$ & $\begin{array}{l}46 \cdot 0 \\
62 \cdot 5 \\
65 \cdot 0 \\
57 \cdot 1 \\
81 \cdot 5 \\
81 \cdot 5 \\
73 \cdot 0 \\
\overline{62 \cdot 5} \\
58 \cdot 5 \\
47 \cdot 0 \\
83 \cdot 0 \\
59 \cdot 5 \\
55 \cdot 0 \\
76 \cdot 2 \\
54 \cdot 5 \\
57 \cdot 5 \\
58 \cdot 0 \\
57 \cdot 0 \\
66 \cdot 5 \\
49 \cdot 5 \\
65 \cdot 5\end{array}$ & $\begin{array}{l}2 \cdot 90 \\
3 \cdot 66 \\
3 \cdot 36 \\
3 \cdot 26 \\
2 \cdot 90 \\
2 \cdot 90 \\
2 \cdot 62 \\
4 \cdot 08 \\
3 \cdot 32 \\
3 \cdot 20 \\
3 \cdot 12 \\
3 \cdot 42 \\
3 \cdot 40 \\
2 \cdot 74 \\
3 \cdot 30 \\
3 \cdot 46 \\
3 \cdot 60 \\
2 \cdot 94 \\
3 \cdot 38 \\
3 \cdot 61 \\
3 \cdot 34 \\
4 \cdot 28 \\
2 \cdot 31\end{array}$ & $\begin{array}{l}\mathbf{F} \\
\mathbf{F} \\
\mathbf{M} \\
\mathbf{F} \\
\mathbf{F} \\
\mathbf{M} \\
\mathbf{F} \\
\mathbf{M} \\
\mathbf{F} \\
\mathbf{M} \\
\mathbf{M} \\
\mathbf{M} \\
\mathbf{F} \\
\mathbf{F} \\
\mathbf{M} \\
\mathbf{F} \\
\mathbf{F} \\
\mathbf{F} \\
\mathbf{M} \\
\mathbf{M} \\
\mathbf{M} \\
\mathbf{F}\end{array}$ & $\begin{array}{l}3 \\
3 \\
3 \\
3 \\
3 \\
3 \\
- \\
3 \\
3 \\
3 \\
1 \\
4 \\
3 \\
3 \\
2 \\
3 \\
3 \\
3 \\
1 \\
3\end{array}$ & $\begin{array}{l}1.40 \\
1.20 \\
1.10 \\
0.90 \\
1.20 \\
2.65 \\
0.32 \\
0.93 \\
0.61 \\
0.55 \\
0.74 \\
0.15 \\
0.38 \\
0.37 \\
0.34 \\
0.79 \\
0.77 \\
2.64 \\
0.65 \\
0.68 \\
1.62 \\
1.19 \\
0.76 \\
0.43\end{array}$ \\
\hline Arith. mean & $38 \cdot 9$ & $40 \cdot 2$ & $62 \cdot 7$ & $3 \cdot 26$ & - & $2 \cdot 8$ & $0.93 \pm 0.64$ \\
\hline
\end{tabular}

Range 0.15-2.65 $\mu \mathrm{g} / \mathrm{g}$ fresh wt. basis

Arith. mean for Jan. $1.41 \mu \mathrm{g} / \mathrm{g}$ fresh wt basis

May $0.72 \mu \mathrm{g} / \mathrm{g}$ fresh wt basis

$-=$ no information.

Sept. $0.89 \mu \mathrm{g} / \mathrm{g}$ fresh wt basis

Table 4 Lead in European placental samples from malformed stillbirths and neonatal deaths

\begin{tabular}{|c|c|c|c|c|c|c|c|c|}
\hline $\begin{array}{l}\text { Delivery } \\
\text { date }\end{array}$ & $\begin{array}{l}\text { Mother's } \\
\text { age }(y)\end{array}$ & $\begin{array}{l}\text { Gestation } \\
\text { period }(w)\end{array}$ & $\begin{array}{l}\text { Mother's } \\
\text { weight }(k g)\end{array}$ & $\begin{array}{l}\text { Child's weight } \\
(\mathrm{kg})\end{array}$ & $\begin{array}{l}\text { Sex of } \\
\text { child }\end{array}$ & $\begin{array}{l}\text { Social class } \\
\text { of father }\end{array}$ & $\begin{array}{l}\text { Nature of } \\
\text { malformation }\end{array}$ & $\begin{array}{l}P b \\
\text { ( } \mu g / g \text { fresh wt } \\
\text { basis) }\end{array}$ \\
\hline 11.2 .71 & 17 & - & - & - & - & 3 & Anencephaly & 1.37 \\
\hline 19.2.71 & - & - & - & - & - & - & $\begin{array}{l}\text { Multiple } \\
\text { malformations }\end{array}$ & 1.51 \\
\hline 11.3.71 & 22 & 42 & $71 \cdot 0$ & 3.43 & $\mathbf{F}$ & 3 & Meningocele & 1.60 \\
\hline 22.3.71 & 30 & 32 & $49 \cdot 0$ & $1 \cdot 10$ & $\mathbf{F}$ & 3 & Anencephaly & 1.74 \\
\hline 17.5 .71 & 23 & 27 & $52 \cdot 0$ & 1.08 & $\mathbf{M}$ & 3 & Microcephaly & 1.07 \\
\hline 19.5.71 & 25 & 35 & 72.0 & $3 \cdot 30$ & $\mathbf{F}$ & 3 & $\begin{array}{l}\text { Persistent ductus } \\
\text { arteriosus }\end{array}$ & 0.53 \\
\hline 1.6 .71 & 26 & 36 & $62 \cdot 0$ & $2 \cdot 67$ & $\mathbf{M}$ & 3 & $\begin{array}{l}\text { Diaphragmatic } \\
\text { hernia }\end{array}$ & 0.64 \\
\hline 15.6 .71 & 24 & 41 & $58 \cdot 0$ & 3.82 & $\mathbf{F}$ & 3 & $\begin{array}{l}\text { Haemangioma } \\
\text { liver }\end{array}$ & 1.65 \\
\hline $\begin{array}{l}29.7 .71 \\
24.9 .71\end{array}$ & $\begin{array}{l}30 \\
25\end{array}$ & $\begin{array}{l}32 \\
39\end{array}$ & $\begin{array}{l}55 \cdot 5 \\
59 \cdot 5\end{array}$ & $\begin{array}{l}1.40 \\
3 \cdot 58\end{array}$ & $\begin{array}{l}\mathbf{M} \\
\mathbf{F}\end{array}$ & $\begin{array}{l}3 \\
4\end{array}$ & $\begin{array}{l}\text { Anencephaly } \\
\text { Extra fingers }\end{array}$ & $\begin{array}{l}0.98 \\
0.82\end{array}$ \\
\hline 18.10 .71 & 23 & 47 & $63 \cdot 7$ & 2.42 & $\mathbf{M}$ & 2 & $\begin{array}{l}\text { Chromosomal } \\
\text { abnormality } \\
\text { (ring D) }\end{array}$ & $2 \cdot 41$ \\
\hline 5.11 .71 & 31 & 40 & $58 \cdot 0$ & 2.06 & $\mathbf{F}$ & 3 & $\begin{array}{l}\text { Chromosomal } \\
\text { abnormality } \\
\text { (trisomy 18) }\end{array}$ & $2 \cdot 41$ \\
\hline 25.11 .71 & 24 & 35 & - & $2 \cdot 50$ & $\mathbf{M}$ & 3 & $\begin{array}{l}\text { Congenital } \\
\text { tumour of CNS }\end{array}$ & $2 \cdot 67$ \\
\hline Arith. mean & $25 \cdot 0$ & $36 \cdot 9$ & $60 \cdot 1$ & 2.49 & - & $3 \cdot 0$ & - & $1.49 \pm 0.69$ \\
\hline
\end{tabular}

Range 0.53-2.67 $\mu \mathrm{g} / \mathrm{g}$ fresh wt basis

$-=$ no information. 
Table 5 Lead in European placental samples from neonatal deaths

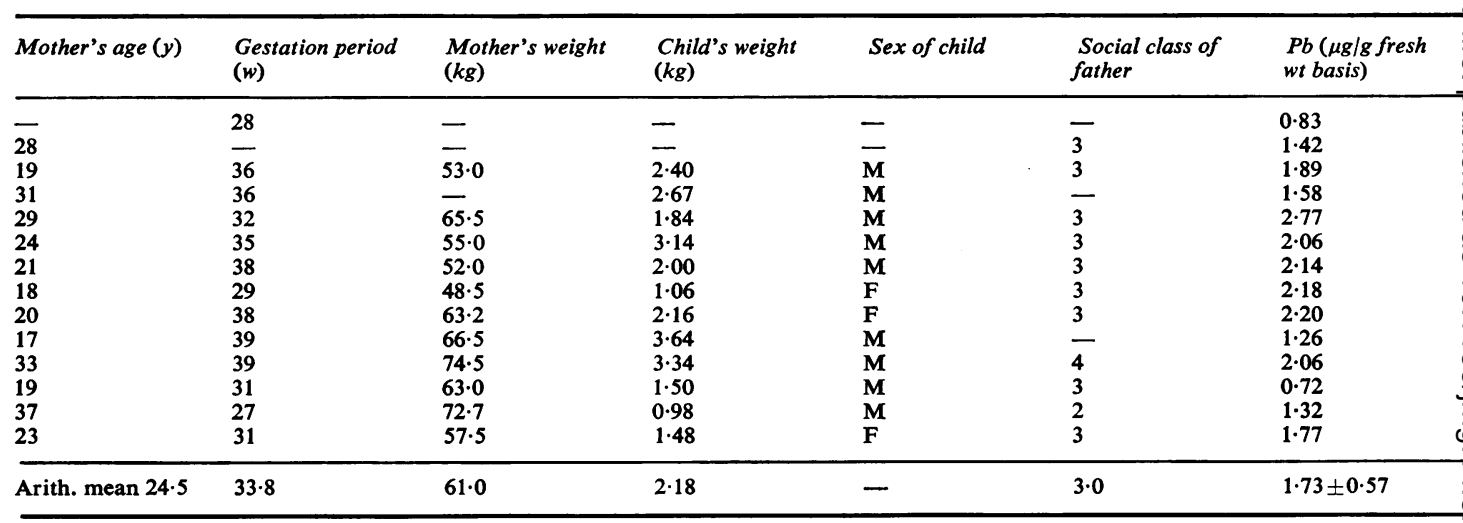

Range $0 \cdot 72-2 \cdot 77 \mu \mathrm{g} / \mathrm{g}$ fresh wt basis

$-=$ no information.

Table 6 Lead in European placental samples from stillbirths without malformations

\begin{tabular}{|c|c|c|c|c|c|c|}
\hline Mother's age $(y)$ & $\begin{array}{l}\text { Gestation period } \\
(w)\end{array}$ & $\begin{array}{l}\text { Mother's weight } \\
\text { (kg) }\end{array}$ & $\begin{array}{l}\text { Child's weight } \\
(\mathrm{kg})\end{array}$ & Sex of child & $\begin{array}{l}\text { Social class of } \\
\text { father }\end{array}$ & $\begin{array}{l}P b(\mu g / g \text { fresh } \\
\text { wt basis) }\end{array}$ \\
\hline 34 & 35 & $52 \cdot 2$ & 1.68 & $\mathbf{F}$ & 3 & $1 \cdot 18^{*}$ \\
\hline 26 & 41 & $61 \cdot 5$ & $3 \cdot 60$ & $\mathbf{M}$ & 3 & 0.61 \\
\hline 25 & 40 & $76 \cdot 0$ & 3.08 & $\mathbf{F}$ & 3 & $0.86^{*}$ \\
\hline 27 & 30 & $82 \cdot 2$ & $1 \cdot 15$ & $\mathbf{F}$ & 3 & $1.55^{*}$ \\
\hline 27 & 33 & - & $1 \cdot 36$ & $\mathbf{M}$ & 4 & $1 \cdot 87^{*}$ \\
\hline - & - & 一 & - & 一 & - & $2 \cdot 19$ \\
\hline 30 & 41 & $50 \cdot 0$ & $2 \cdot 75$ & $\mathbf{F}$ & 3 & $1 \cdot 81$ \\
\hline 20 & 41 & $62 \cdot 0$ & $2 \cdot 80$ & $\mathbf{F}$ & - & $1 \cdot 56$ \\
\hline 30 & 34 & $68 \cdot 5$ & 1.40 & $\mathbf{M}$ & 3 & $1 \cdot 42 *$ \\
\hline Arith. mean 27.4 & 36.9 & $64 \cdot 6$ & $2 \cdot 23$ & - & $3 \cdot 1$ & $1.45 \pm 0.50$ \\
\hline
\end{tabular}

Range 0.61-2.19 $\mu \mathrm{g} / \mathrm{g}$ fresh wt basis - = no information.

*Macerated.

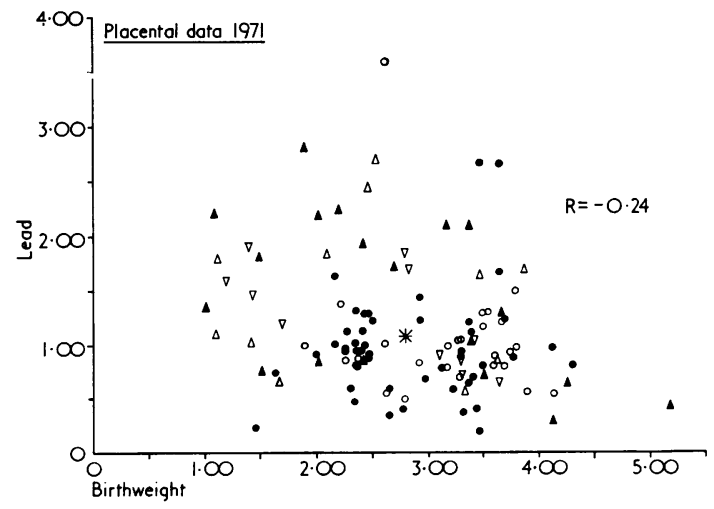

Fig. 1 Distribution of lead levels by birthweight for stillbirths $(\nabla)$, neonatal deaths $(\mathbf{\Lambda})$, malformed stillbirths and neonatal deaths, $(\triangle)$ and surviving births $(\bullet$ European and $\bigcirc$ Indian). The correlation coefficient refers to the non-immigrant surviving births.

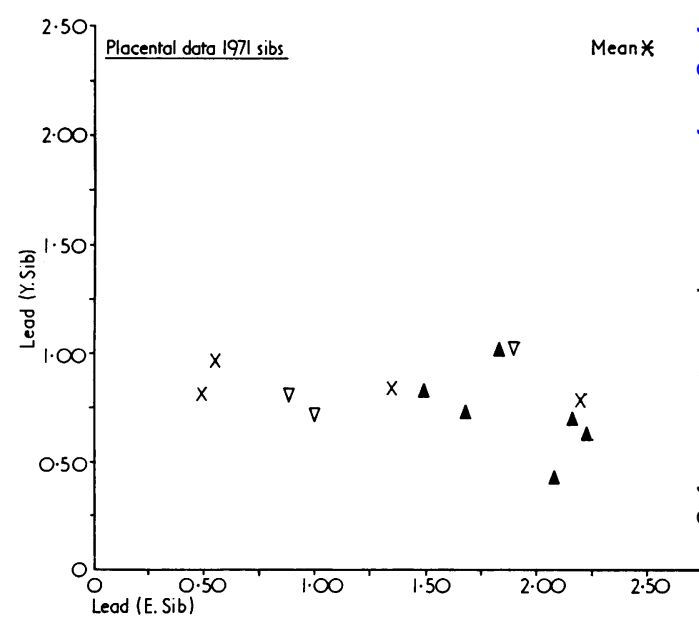

Fig. 2 Distribution of lead levels in normal sibs to Indian births $(x)$ and to European stillbirths $(\nabla)$ and neonatal deaths $(\mathbf{\Delta})$. 
Table 7 Placental lead in sibs of 1971 samples

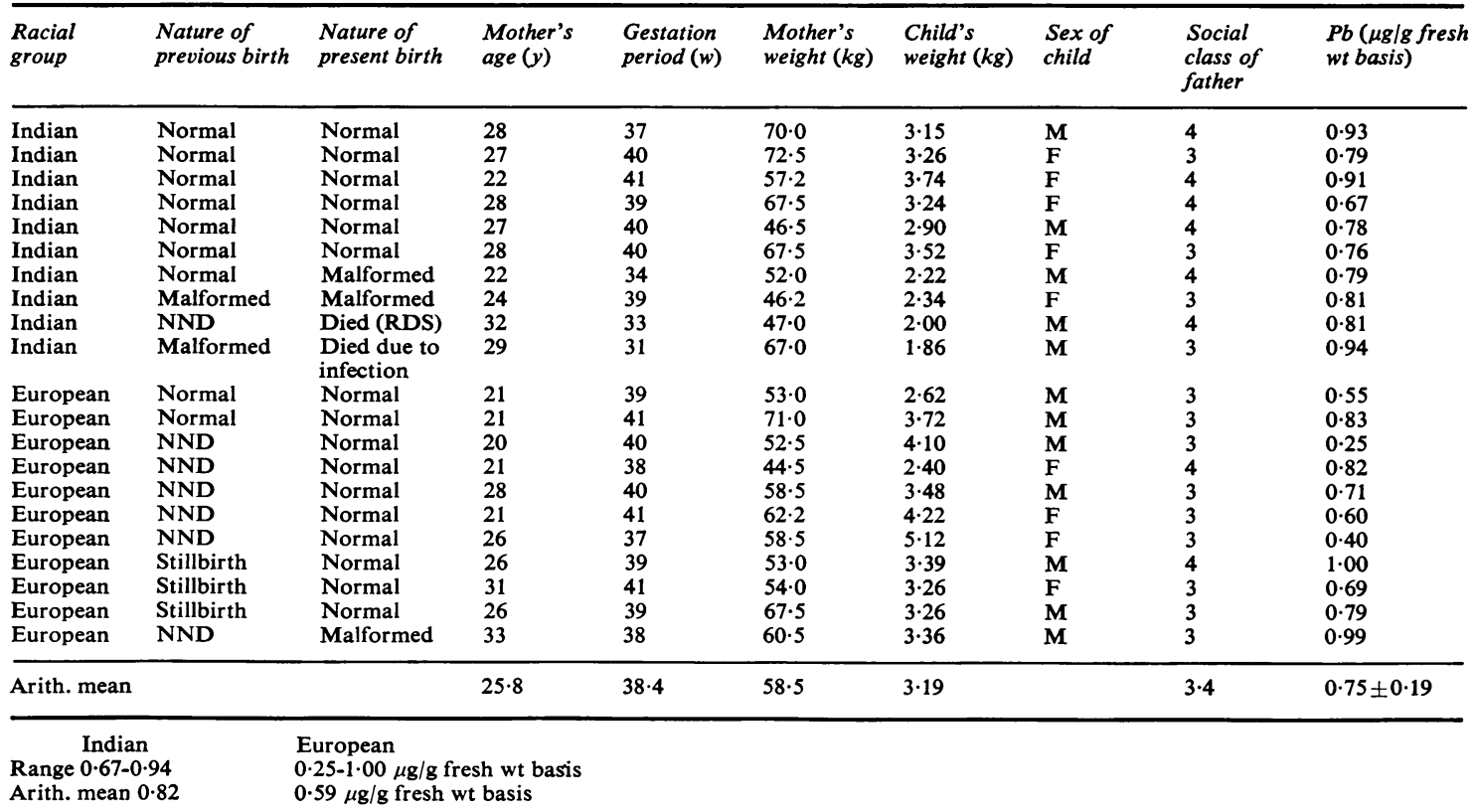

Table 8 Placental lead in European premature birth samples

\begin{tabular}{|c|c|c|c|c|c|c|}
\hline $\begin{array}{l}\text { Mother's age } \\
\text { (y) }\end{array}$ & $\begin{array}{l}\text { Gestation period } \\
(w)\end{array}$ & $\begin{array}{l}\text { Mother's weight } \\
(\mathrm{kg})\end{array}$ & $\begin{array}{l}\text { Child's weight } \\
(\mathrm{kg})\end{array}$ & Sex of child & $\begin{array}{l}\text { Social class of } \\
\text { father }\end{array}$ & $\begin{array}{l}P b(\mu g / g \text { fresh } \\
\text { wt basis })\end{array}$ \\
\hline 23 & 39 & 44.5 & $2 \cdot 34$ & $\mathbf{M}$ & 2 & 0.75 \\
\hline 28 & 30 & - & 1.62 & $\mathrm{~F}$ & 5 & 0.70 \\
\hline 28 & 40 & 77.0 & $2 \cdot 12$ & $\mathbf{M}$ & 4 & 1.59 \\
\hline 24 & 34 & $59 \cdot 5$ & $2 \cdot 23$ & $\mathbf{F}$ & 1 & 1.09 \\
\hline 31 & 37 & $68 \cdot 0$ & 2.40 & $\mathbf{M}$ & 3 & 0.96 \\
\hline 23 & 39 & $53 \cdot 0$ & $2 \cdot 22$ & $\mathbf{F}$ & - & 0.90 \\
\hline 33 & 40 & - & $2 \cdot 30$ & $\mathbf{M}$ & 1 & 0.98 \\
\hline 27 & 30 & $57 \cdot 0$ & 1.43 & $\mathbf{M}$ & 3 & 0.21 \\
\hline 30 & 40 & $58 \cdot 2$ & 2.42 & $\mathbf{F}$ & 3 & 1.25 \\
\hline 31 & 37 & 59.5 & $2 \cdot 48$ & $\mathbf{F}$ & - & $1 \cdot 19$ \\
\hline 27 & 40 & $61 \cdot 0$ & $2 \cdot 26$ & $\mathbf{M}$ & 4 & 0.55 \\
\hline 25 & 39 & $49 \cdot 0$ & $2 \cdot 22$ & $\mathbf{F}$ & 3 & 0.92 \\
\hline 24 & 42 & $49 \cdot 0$ & $2 \cdot 42$ & $\mathbf{M}$ & 4 & 1.25 \\
\hline 38 & 33 & $47 \cdot 5$ & 1.98 & M & 3 & 0.88 \\
\hline 33 & 36 & $59 \cdot 0$ & $2 \cdot 32$ & $\mathbf{M}$ & 4 & 0.91 \\
\hline 32 & 37 & 62.5 & $2 \cdot 32$ & $\mathrm{~F}$ & 3 & 0.76 \\
\hline 27 & 35 & $51 \cdot 0$ & $2 \cdot 40$ & $\mathbf{M}$ & - & 1.25 \\
\hline 27 & 38 & $54 \cdot 5$ & 2.42 & $\mathbf{F}$ & 3 & 0.84 \\
\hline 27 & 40 & $69 \cdot 5$ & 2.32 & $\mathbf{F}$ & 3 & 1.29 \\
\hline 28 & 37 & 57.0 & $2 \cdot 34$ & $\mathbf{M}$ & 2 & 0.85 \\
\hline 32 & 39 & $54 \cdot 5$ & $2 \cdot 37$ & $\mathbf{M}$ & 2 & 0.91 \\
\hline 27 & - & - & 2.43 & - & - & 0.87 \\
\hline 23 & - & - & $2 \cdot 38$ & - & - & $1 \cdot 11$ \\
\hline 27 & - & - & $2 \cdot 12$ & - & - & 0.97 \\
\hline Arith. mean $28 \cdot 1$ & $37 \cdot 2$ & $57 \cdot 4$ & $2 \cdot 24$ & & 2.9 & $0.96 \pm 0.28$ \\
\hline
\end{tabular}

Range 0.21-1.59 $\mu \mathrm{g} / \mathrm{g}$ fresh wt. 
Table 9 Summary of placental lead results

\begin{tabular}{ll}
\hline Nature of samples & $\begin{array}{l}P b \\
(\mu g / g \text { fresh } \text { wt basis })\end{array}$ \\
\hline Normal Indian samples & $1.13 \pm 0.68$ \\
European samples from & \\
(a) Normal births & $0.93 \pm 0.64$ \\
(b) Normal premature births & $0.96 \pm 0.28$ \\
(c) Malformed stillbirths and neonatal & $1.48 \pm 0.69$ \\
deaths & $1.73 \pm 0.57$ \\
(d) Neonatal deaths & $1.45 \pm 0.50$ \\
(e) Stillbirths & $0.75 \pm 0.19$ \\
\hline
\end{tabular}

collected together in Table 9 and expressed graphically in Figs. 1 and 2.

\section{Discussion}

Our first conclusion at this state is that the results appear to be significant and to justify further work. In particular we wish to examine other groups such as Asian samples where stillbirth or neonatal death has resulted. Further work concerning the background of the mother is also required. Just as blood-lead levels increase with urban environment (Waldron and Stofën, 1974) it is obviously possible that placental lead levels could show some variation within the Birmingham environment, and Thieme et al. (1974) have shown that placental lead levels were higher for mothers in dense urban areas. A previous bloodlevel survey in Birmingham by Betts et al. (1973) has implicated 'Surma' as a possible reason for higher Asian blood lead levels and in another family study the blood lead level of an Asian mother who did use 'Surma' was found to be higher than that of a father who did not (Warley et al., 1968). However, the Indian placental lead levels were not particularly high: in a fairly random survey of Indian households about a third of women used 'Surma', which is mostly lead sulphide $(75 \% \mathrm{PbS})$. The higher placental lead levels in the stillbirths and neonatal deaths themselves of course do not prove that current environmental lead levels are having any adverse effect on the fetus. It could be argued at this stage that placental lead is an environmental indicator rather than an environmental hazard and as such its correlation with the other well-known environmental indicators such as social class and home environment should be investigated further.

In preliminary work which we have carried out on placental specimens from Stoke-on-Trent where some of the mothers were working in the pottery industry no high values were obtained and mean values were only half those found in similar Birmingham samples.

If substantiated by further work, our findings concerning placental lead levels in stillbirths and neonatal births are remarkable. In only $7 \%$ of the normal births were placental lead levels greater tha $1.5 \mu \mathrm{g} / \mathrm{g}$ whereas $61 \%$ of the stillbirths or neonata deaths had levels greater than this. This does not of course mean that lead must be a causal factor in suct deaths but it certainly suggests the necessity for further lead analyses on stillbirth tissues. The alternatives explanation, that lead accumulates in times of fet stress, is one which the present evidence appears to support. For an assessment of this proposal we have planned further work which will include in addition the analyses of maternal tisues or fluids.

We are grateful to Mr Robert Lancaster and Mer Jagdish Parekh for writing computer programmes and Miss Helena Haimila for technical assistance. Wo are also grateful to the Department of Health ancep Social Security and to the Environmental Research Programme of the European Communities (Re search Contract No: 8304) for financial help.

\section{References}

Baglan, R. J., Brill, A. B., Schulert, A., Wilson, D., Larseñ K., Dyer, N., Mansour, M., Schaffner, L., Hoffman, $\mathbf{L}_{\oplus}^{-}$ and Davies J. (1974). Utility of placental tissue as an in dicator of trace element exposure to adult and fetus. Environmental Research, 8, 64-70.

Bakir, F., Damluji, L., Amin-Zaki, M., Murtadha, MP Khalidi, A., Al-Rawi, N. Y., Tikriti, S., Dhahir, H. I Clarkson, T. W., Smith, J. C., and Doherty, R. A. (1973) Methylmercury poisoning in Iraq. Science, 181, 230-241.

Barlow, P. J., and Khera, A. K. (1975). Sample preparatiof using tissue solubilization by Soluene- $350^{\mathrm{TM}}$ for lead determinations by graphite furnace atomic absorptio spectrophotometry. Atomic Absorption Newsletter (U.S.A.尹 14, 149-150.

Betts, P. R., Astley, R., and Raine, D. N. (1973). Lead in toxication in children in Birmingham. British Medicä Journal, 1, 402-406.

Cantarow, A., and Trumper, M. (1944). Lead Poisoning Williams and Wilkins, Baltimore.

Collucci, A. V., Hammer, D. I., Williams, M. E., Hinners T. A., Pinkerton, C., Kent, J. L., and Love, G. J. (1973內 Pollutant burdens and biological response. Archives Environmental Health, 27, 151-154.

Dawson, E. B., Croft, H. A., Clark, R. R., and McGanitQ W. J. (1968). Study of seasonal variations in nine cations of normal term placentas. American Journal of Obstetries and Gynecology, 92, 354-361.

Einbrodt, H. J., Schiereck, F. W., and Kinny, H. (1973̄ Uber die Ablagerung von Blei in den Verkalkungen deP menschlichen Placenta. Archiv für Gynakologie, 213, 303 306.

Ferm, V. H. (1969). The synteratogenic effect of lead and cadmium. Experientia, 25, 56-57.

Ferm, V. H., and Carpenter, S. J. (1967). Developmenta malformations resulting from the administration of lead salts. Experimental Molecular Pathology, 7, 208-213.

Holberg, R. E., and Ferm, V. H. (1969). Interrelationships of selenium, cadmium and arsenic in mammalian terato $D$ genesis. Archives of Environmental Health, 18, 873-877.

Horiuchi, K., Horiguchi, S., and Suekane, M. (1959). Studie on the industrial lead poisoning. Osaka City Medic of Journal, 5, 41-70. 
Khera, A. K., and Wibberley, D. G. (1976). Some analytical problems concerning trace-metal analysis in human placentae. Proceedings of the Analytical Division of the Chemical Society (U.K.), 13, 340-342.

Lanzola, E., Allegrini, M., and Breuer, F. (1973). Lead levels in infant food sold on the Italian market. In Proceedings of the International Symposium on Environmental Health Aspects of Lead, pp. 333-44. Commission of European Communities, Luxembourg.

Matsumota, H., Koga, G., and Takeuchi, T. (1965). Fetal minnimata disease. Journal of Neuropathology and Experimental Neurology, 24, 563-574.

Suzuki, T., Miyama, T., and Katsunuma, H. (1971). Comparison of mercury contents in maternal blood, umbilical cord blood and placental tissues. Bulletin of Environmental Contamination and Toxicology, 5, 502-508.

Thieme, R., Schramel, P., Klose, B. J., and Waidl. E. (1974).
Der EinfluBregionales Umweltfaktoren auf die Spurenelement-zusammensetzung der Plazenta. Geburtshilfe und Frauenheilkunde, 34, 36-41.

Thürauf, J., Schaller, K. H., Engelhardt E., and Gossler, K. (1975). Der Kadmium-Gehalt der menschlichen Placenta. International Archives of Occupational And Environmental Health, 36, 19-27.

Waldron, H. A., and Stofën, D. (1974). Sub-clinical Lead Poisoning, p. 117. Academic Press, London.

Warley, M. A., Blackledge, P., and O'Gorman, P. (1968). Lead poisoning from eye cosmetic. British Medical Journal, $1,117$.

Requests for reprints to Professor D. G. Wibberley, Department of Pharmacy, University of Aston in Birmingham, Gosta Green, Birmingham B4 7ET. 